\title{
MiniAp-4: A Venom-Inspired Peptidomimetic for Brain Delivery
}

\author{
Benjamí Oller-Salvia, Macarena Sánchez-Navarro, Sonia Ciudad, Marc Guiu, Pol Arranz- \\ Gibert, Cristina Garcia, Roger R. Gomis, Roméo Cecchelli, Jesús García, Ernest Giralt,* and \\ Meritxell Teixidó*
}

\begin{abstract}
Drug delivery across the blood-brain barrier (BBB) is a formidable challenge for therapies targeting the central nervous system. Although $B B B$ shuttle peptides enhance transport into the brain non-invasively, their application is partly limited by lability to proteases. The present study proposes the use of cyclic peptides derived from venoms as an affordable way to circumvent this drawback. Apamin, a neurotoxin from bee venom, was minimized by reducing its complexity, toxicity, and immunogenicity, while preserving brain targeting, active transport, and protease resistance. Among the analogues designed, the monocyclic lactambridged peptidomimetic MiniAp-4 was the most permeable. This molecule is capable of translocating proteins and nanoparticles in a human-cell-based BBB model. Furthermore, MiniAp-4 can efficiently deliver a cargo across the $B B B$ into the brain parenchyma of mice.
\end{abstract}

The incidence of brain diseases is increasing mainly because of population aging. Unfortunately, most molecules intended for therapy and with high in vitro efficiency fail to reach their targets because they are unable to cross the blood-brain barrier (BBB) in adequate amounts. ${ }^{[1]}$ One of the most promising non-invasive strategies under investigation for drug delivery to the central nervous system (CNS) is the use of BBB shuttles. ${ }^{[2]}$ These vectors are able to enhance the passage of compounds across the $\mathrm{BBB}$ without altering its integrity. In

[*] Dr. B. Oller-Salvia, Dr. M. Sánchez-Navarro, S. Ciudad, M. Guiu,

P. Arranz-Gibert, C. Garcia, Dr. R. R. Gomis, Dr. J. García,

Prof. E. Giralt, Dr. M. Teixidó

Institute for Research in Biomedicine (IRB Barcelona)

The Barcelona Institute of Science and Technology

Baldiri Reixac, 10, 08028 Barcelona (Spain)

E-mail: ernest.giralt@irbbarcelona.org meritxell.teixido@irbbarcelona.org

Dr. R. R. Gomis

ICREA (Spain)

Prof. R. Cecchelli

University of Artois, Faculté Jean Perrin

Jean Souvraz-SP 18, 62307 Lens (France)

Prof. E. Giralt

University of Barcelona

Department of Organic Chemistry (Spain)

(-) Supporting information for this article is available on the WWW under http://dx.doi.org/10.1002/anie.201508445.

(C) 2015 The Authors. Published by Wiley-VCH Verlag GmbH \& Co. $\mathrm{KGaA}$. This is an open access article under the terms of the Creative Commons Attribution Non-Commercial NoDerivs License, which permits use and distribution in any medium, provided the original work is properly cited, the use is non-commercial and no modifications or adaptations are made. the last few years, much research effort has been devoted to identifying peptide shuttles because these molecules are more economical, less immunogenic, and have higher chemical versatility than large proteins, such as Trojan horse antibodies. Nevertheless, an important drawback of existing sequences is their high lability to serum proteases. We and others have recently shown that peptides with reversed sequences and Damino acids (retro-enantio approach) can be more efficient carriers than their L-counterparts. ${ }^{[3]}$ Nonetheless, this strategy is relatively costly and may decrease the affinity of the peptide for the receptor that mediates its transport. A way to circumvent these drawbacks could be the use of natural cyclic peptides targeting the CNS, such as those found in venoms. However, an inconvenience of using these compounds as BBB shuttles is their high toxicity. In particular, the bicyclic peptide apamin, found in bee venom, crosses the BBB and blocks calcium-mediated potassium channels. ${ }^{[4]}$ Despite the proven CNS-targeting capacity of apamin, ${ }^{[5]}$ the extended application of this molecule has been limited by its toxicity, high immunogenicity, ${ }^{[6]}$ and relatively complex structure. Two years ago, we reported that the replacement of arginines by ornithines yielded a non-toxic apamin analogue that is able to cross a tight monolayer of bovine endothelial cells mimicking the BBB ${ }^{[7]}$ Our present study aimed to obtain all-L-proteaseresistant shuttles by minimizing apamin, while reducing its toxicity and immunogenicity. Furthermore, we studied the ability of these shuttles to carry large cargoes in a human-cellbased BBB model and to reach the mouse brain parenchyma.

To simplify the structure of apamin, we first synthesized the native peptide and a non-reported analogue that does not contain the main residues responsible for its toxicity (MiniAp-1; Figure 1a). We assayed both compounds in a bovine-cell-based BBB model and found that the permeability of MiniAp-1 was $60 \%$ higher than that of apamin (Figure 1b; see also the Supporting Information, Table S2). Lucifer yellow was used as an integrity marker. ${ }^{[8]}$ The strong inhibition of MiniAp-1 transcytosis by a change in temperature or the addition of sodium azide (Figure S2a) indicated that the shuttle was transported mainly through an active mechanism. Next, we showed that this analogue was capable of carrying small cargoes, such as dopamine, carboxyfluorescein $(\mathrm{cF})$, sulforhodamine $\mathrm{B}$, and an amyloid $\beta$-sheet breaker peptide (Figure S2b). These results encouraged us to further dissect the structure and prepare minimized derivatives based on the loop between Cys-3 and Cys-11. We first synthesized a linear version (MiniAp-2), which was transported less efficiently than MiniAp-1 and readily degraded by serum proteases (Figure 1c). We then prepared a monocyclic analogue by connecting Cys-3 and Cys-11 
a)

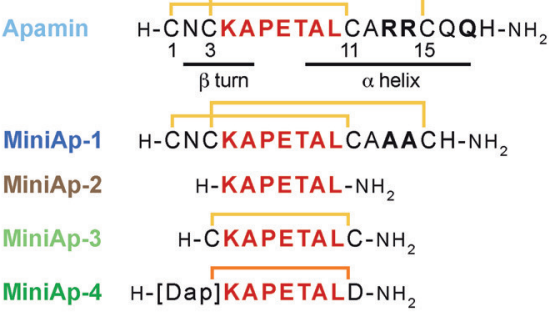

b)

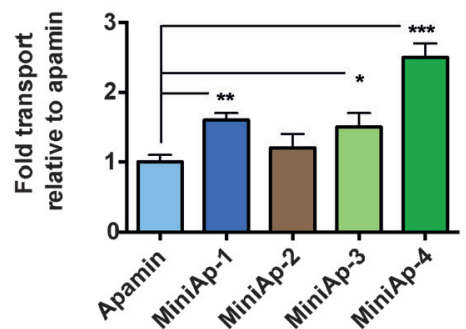

c)

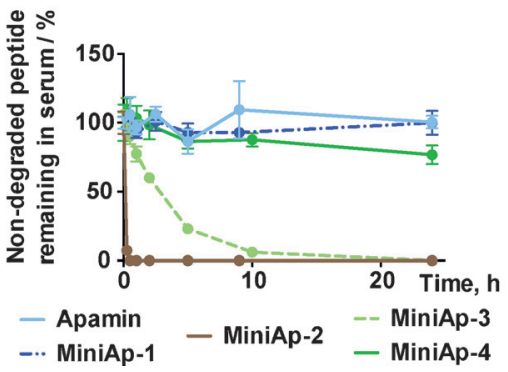

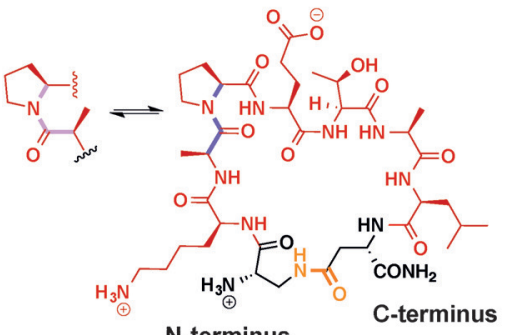

$\mathrm{N}$-terminus

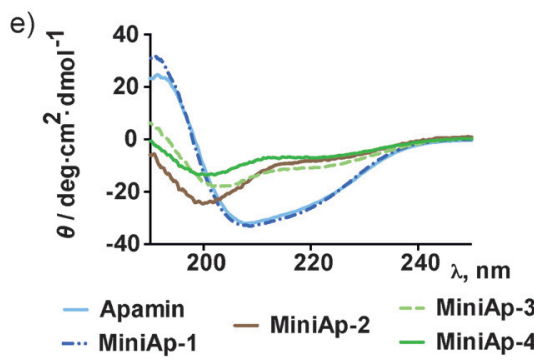

f)

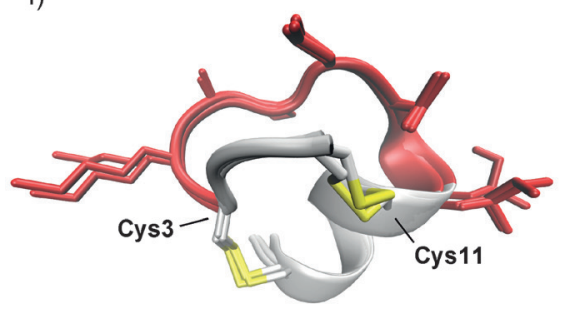

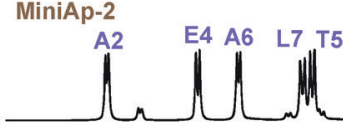

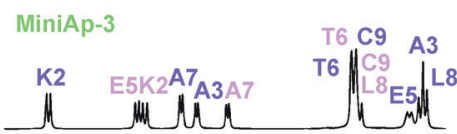

MiniAp-4

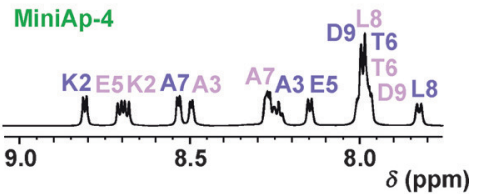

h)
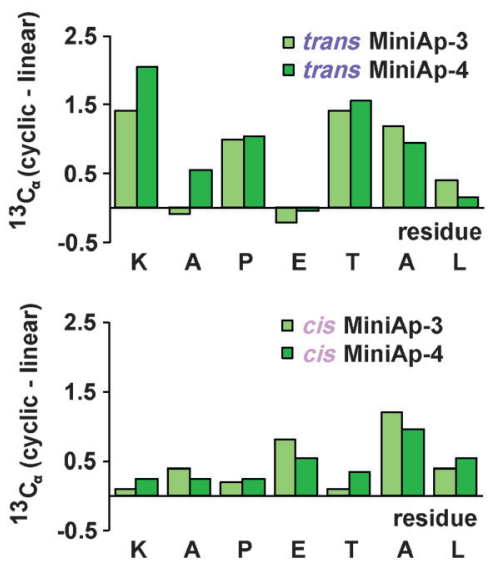

Figure 1. Comparison of mini-apamin shuttle candidates. a) Peptide sequences. The disulfides are depicted in yellow and the lactam bridge in orange. b) Relative transport of peptides in the bovine-cell-based model. c) Stability of the peptides in human serum. d) Molecular representation of MiniAp-4, showing the equilibrium between the trans and cis Ala-Pro conformations. e) CD spectra. f) The three lowest-energy NMR structures of MiniAp-1. g) Amide regions of the ${ }^{1} \mathrm{H}$ NMR spectra of MiniAp-2, MiniAp-3, and MiniAp-4. The amide resonances of the trans (purple) and cis (pink) conformers are labeled. h) Comparison of the ${ }^{13} \mathrm{C}_{\alpha}$ chemical shift values of the linear derivative (MiniAp-2) and the trans (top) and cis (bottom) conformers of the cyclic analogues (MiniAp-3 and MiniAp-4). In Figure $1 \mathrm{~b}$ and c, error bars represent the standard error of the mean (SEM; $n=3, * p<0.05, * * p<0.01$, *** $p<0.001)$.

(MiniAp-3). This peptide showed permeability values similar to those of MiniAp-1, and was over 30 times more resistant to serum proteases than the linear version. Finally, by switching the disulfide to a lactam bridge, we obtained MiniAp-4 (Figure 1d). Remarkably, the permeability of the latter was $50 \%$ higher than that of MiniAp-3, which could be attributable to its impressive resistance to serum proteases $\left(t_{1 / 2}=\right.$ $24 \mathrm{~h})$.

Aiming to further explain the differences in BBB transport between the analogues, we studied their conformational preferences. The $\alpha$-helical structure of MiniAp-1, typical of apamin, ${ }^{[9]}$ was confirmed by circular dichroism (CD; Figure $1 \mathrm{e}$ ) and by the following NMR parameters: ${ }^{1} \mathrm{H}_{\alpha}$ and ${ }^{13} \mathrm{C}_{\alpha}$ secondary chemical shifts, NOE patterns, the NH temperature coefficients, and the ${ }^{3} J_{\alpha \mathrm{NH}}$ coupling constants (Tables S3, S4 and Figure S5). The three-dimensional structures of this analogue were determined by simulated annealing methods (Figure $1 \mathrm{f}$ and Table S5), whereas the other peptides were too flexible to provide a reliable model. In particular, the CD spectrum and the NMR parameters of the linear analogue (MiniAp-2) showed a clear random coil behavior. Although the structural data for MiniAp-3 and MiniAp-4 suggested that they had no defined secondary structure, a larger amide proton chemical shift dispersion (Figure $1 \mathrm{~g}$ ) and notable differences in the ${ }^{13} \mathrm{C}_{\alpha}$ chemical shifts (Figure $1 \mathrm{~h}$ ) indicated that they had lower conformational flexibility than MiniAp-2. Most remarkably, whereas the linear analogue showed a 1:9 cis/trans Ala-Pro conformer population ratio like the bicyclic peptides (apamin and MiniAp-1), the two monocyclic derivatives showed a ratio of 1:1. The higher preference for the $c i s$ Ala-Pro rotamer in MiniAp-4 with respect to MiniAp-1 could partly account for the difference in permeability between the two analogues with highest resistance to proteases.

We next studied the toxicity and immunogenicity of MiniAp-1 and MiniAp-4. A remarkable decrease in acute toxicity for both analogues was confirmed through intravenous injection in mice at a dose 24 times the $\mathrm{LD}_{50}$ of apamin $^{[10]}$ causing no observable symptoms during 24 hours. Regarding immunogenicity, in a twelve week study, we confirmed that apamin was highly immunogenic, even when injected at $25 \mu \mathrm{g}$ doses to avoid toxicity. However, although MiniAp-1 generated six-fold fewer IgGs than apamin, it was still relatively virulent (Figure 2). Conversely, the amount of antibodies generated by MiniAp-4 was at least three orders of magnitude lower than that produced by apamin. 
a)

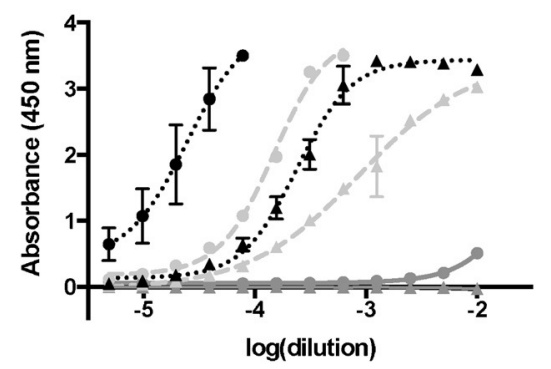

... Apamin

- MiniAp-1

- MiniAp-4

- After 4 doses

- After 7 doses

b)

\begin{tabular}{|c|ccc|}
\hline & Apamin & MiniAp-1 & MiniAp-4 \\
\cline { 1 - 4 } After 4 doses & $3.6 \pm 0.1$ & $3.0 \pm 0.1$ & $<<2$ \\
\cline { 1 - 3 } After 7 doses & $4.6 \pm 0.2$ & $3.8 \pm 0.1$ & $<2$ \\
\hline
\end{tabular}

Figure 2. IgG titrations. a) Absorbance in the ELISA plate versus serum dilution. b) Antibody titer reported as $-\log \left(E C_{50}\right) \cdot n=4$ for apamin and MiniAp-4, $n=2$ for MiniAp-1. Error bars represent the standard deviation.

Taking into account the results from the permeability, stability, and immunogenicity experiments, we determined that MiniAp-4 was the lead mini-apamin shuttle candidate. To assess whether the peptide was suitable to transport large cargoes, we studied the internalization mechanism of the cFMiniAp- 4 construct in bEnd. 3 cells using inhibitors of the two main pathways reported to be involved in transcytosis. Cell uptake was not affected by chlorpromazine, which inhibits internalization through clathrin-coated pits, ${ }^{[11]}$ but was reduced by filipin (Figure S3), which interferes with caveolaemediated endocytosis. ${ }^{[12]}$

At this point, we switched to a more relevant human-cellbased $\mathrm{BBB}$ model, which has recently been developed to study transcytosis and has good correlation with human clinical data. ${ }^{[13]}$ In the new model, MiniAp-4 showed greater permeability than in the bovine one (Table S2). This observation is highly relevant for the potential translation of the shuttle to humans as it suggests that the translocation mechanism is also operating in human cells. The ability of MiniAp-4 to carry covalently attached macromolecules and nanoparticles was first confirmed by a 1.6-fold increase in the transport of GFP (Figure 3a,b). Importantly, a scrambled version of the peptide did improve neither the permeability nor the cell uptake of GFP (Figure S9). These results underscore the relevance of the residue sequence in MiniAp-4 to mediate translocation and would be consistent with a receptor-mediated transcytosis mechanism. Furthermore, a twofold transport increase was observed for far-red imaging quantum dots (Qdot-605). This enhancement is similar to those achieved with similar cargoes using other peptide BBB shuttles. ${ }^{[14]}$ However, in contrast to most reported shuttles, MiniAp-4 is small and resistant to protease degradation, properties that are considerable advantages for production and in vivo efficiency, respectively. Finally, we assayed $12 \mathrm{~nm}$ gold nanoparticles (AuNPs) because of their potential therapeutic applications. ${ }^{[15]}$ In this case, the increase was even more pronounced (over 20-fold), probably because of the low permeability of the cargo alone and the higher functionalization (Figure $3 \mathrm{a}$ ).

After finding that MiniAp-4 transports various compounds in the human BBB model, we demonstrated that it was capable of delivering a cargo in mice. Brain accumulation of the peptide linked to cyanine 5.5 (Cy5.5-MiniAp-4) was compared to the same fluorophore capped with cysteamine (Cy5.5-CA; Figure S10). Upon intravenous injection of the compounds, a 4.3 -fold increase of fluorescence in the brain a)

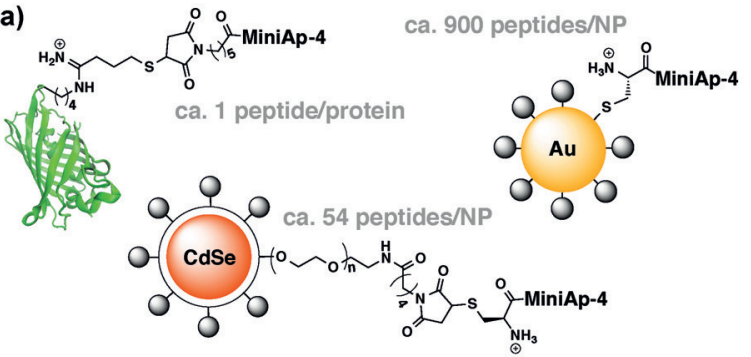

b)

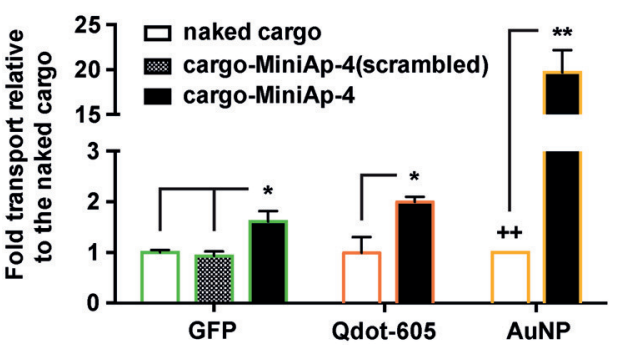

c)

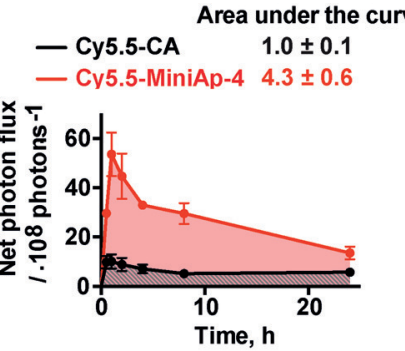

d)

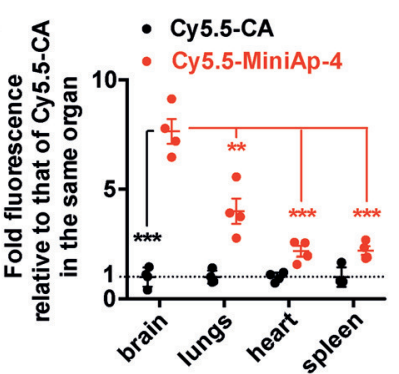

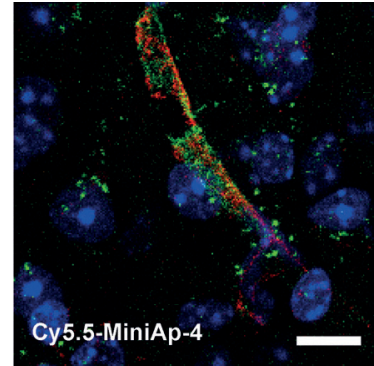

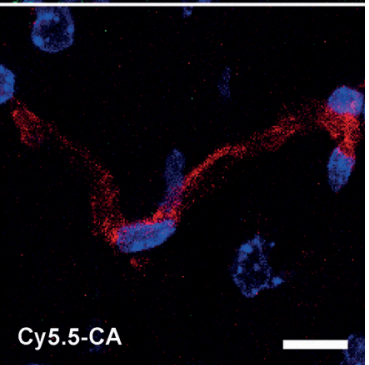

Figure 3. MiniAp-4 as a new BBB shuttle. a) Protein and NP shuttle conjugates. b) Permeability enhancements that are due to MiniAp-4 in the human-cell-based BBB model. c) Fluorescence intensity of the brain region measured in vivo. d) Fluorescence intensity of various organs ex vivo. e) Representative confocal microscopy images of brain slices (cortex) of mice injected with Cy5.5-MiniAp-4 (top) and Cy5.5-CA (bottom). The Cy5.5 conjugates are shown in green, capillaries in red, and cell nuclei in blue. Scale bars: $10 \mu \mathrm{m}$. Error bars represent the SEM ( $n=3$, * $p<0.05$, $* * p<0.01, * * * p<0.001) .++$ indicates the quantification limit. 
region was observed (Figure $3 \mathrm{c}$ ). The enhancement provided by MiniAp-4 was even higher (7.6-fold) when measured in the excised brains upon perfusion with PBS $1 \mathrm{~h}$ after injection and was significantly selective for the brain compared to the lungs, heart, and spleen (Figures $3 \mathrm{~d}$ and S11).

Finally, using fluorescence microscopy, we observed that the targeted fluorophore not only colocalized with capillaries but also was found in the form of aggregates in the brain parenchyma, unlike the cysteamine version (Figure $3 \mathrm{e}$ ). The aggregates were found inside neurons, a highly relevant observation for the potential treatment of neurodegenerative diseases, and only colocalized to a small extent with a glial marker (Figure S12).

In summary, we have shown that MiniAp-4, a minimized and more permeable peptidomimetic version of apamin, is resistant to proteases and able to efficiently deliver diverse cargoes across the blood-brain barrier in a human-cell-based model and also in vivo. Furthermore, this shuttle has a remarkable brain-targeting capacity and is considerably less toxic and immunogenic than the native peptide.

\section{Acknowledgements}

We thank Josep Calderón, Roger Prades, Emmanuel Sevin, and Nuno Vasconcelos for their help with the ICP-MS measurements, the bovine $\mathrm{BBB}$ model, the human $\mathrm{BBB}$ model, and mice perfusion, respectively. We also acknowledge the NMR facility at CCiT UB. This study was funded by MINECO-FEDER (Bio2013-40716-R), MICINN (SAF201346196), RecerCaixa-2014-Gate2Brain, and the Generalitat de Catalunya (X.R.B.; 2009SGR-1005, 2014SGR-535, PROVAT2011-013). B.O.-S. and M.S.-N. hold "La Caixa"/IRB Barcelona and Juan de la Cierva fellowships, respectively.

Keywords: apamin · blood-brain barrier · drug delivery . peptides

How to cite: Angew. Chem. Int. Ed. 2016, 55, 572-575 Angew. Chem. 2016, 128, 582-585
[1] W. M. Pardridge, J. Cereb. Blood Flow Metab. 2012, 32, 19591972.

[2] M. Malakoutikhah, M. Teixidó, E. Giralt, Angew. Chem. Int. Ed. 2011, 50, 7998-8014; Angew. Chem. 2011, 123, 8148-8165.

[3] a) X. Wei, C. Zhan, X. Chen, J. Hou, C. Xie, W. Lu, Mol. Pharm. 2014, 11, 3261-3268; b) R. Prades, B. Oller-Salvia, S. M. Schwarzmaier, J. Selva, M. Moros, M. Balbi, V. Grazú, J. M. de La Fuente, G. Egea, N. Plesnila, M. Teixidó, E. Giralt, Angew. Chem. Int. Ed. 2015, 54, 3967-3972; Angew. Chem. 2015, 127, 4039-4044; c) X. Wei, C. Zhan, Q. Shen, W. Fu, C. Xie, J. Gao, C. Peng, P. Zheng, W. Lu, Angew. Chem. Int. Ed. 2015, 54, $3023-$ 3027; Angew. Chem. 2015, 127, 3066-3070.

[4] C. Labbé-Jullié, C. Granier, F. Albericio, M. L. Defendini, B. Ceard, H. Rochat, J. van Rietschoten, Eur. J. Biochem. 1991, 196, 639-645.

[5] J. Wu, H. Jiang, Q. Bi, Q. Luo, J. Li, Y. Zhang, Z. Chen, C. Li, Mol. Pharm. 2014, 11, 3210-3222.

[6] M. L. Defendini, M. Pierres, A. Regnier-Vigouroux, H. Rochat, C. Granier, Mol. Immunol. 1990, 27, 551-558.

[7] B. Oller-Salvia, M. Teixidó, E. Giralt, Biopolymers 2013, 100, $675-686$.

[8] M. Culot, S. Lundquist, D. Vanuxeem, S. Nion, C. Landry, Y. Delplace, M. P. Dehouck, V. Berezowski, L. Fenart, R. Cecchelli, Toxicol. In Vitro 2008, 22, 799-811.

[9] S. Fiori, S. Pegoraro, S. Rudolph-Böhner, J. Cramer, L. Moroder, Biopolymers 2000, 53, 550-564.

[10] W. L. Cosand, R. B. Merrifield, Proc. Natl. Acad. Sci. USA 1977, $74,2771-2775$.

[11] J. E. Schnitzer, P. Oh, E. Pinney, J. Allard, J. Cell Biol. 1994, 127, $1217-1232$.

[12] A. Subtil, A. Hémar, A. Dautry-Varsat, J. Cell Sci. 1994, 107, $3461-3468$.

[13] R. Cecchelli, S. Aday, E. Sevin, C. Almeida, M. Culot, L. Dehouck, C. Coisne, B. Engelhardt, M. P. Dehouck, L. Ferreira, PLOS ONE 2014, 9, e99733.

[14] a) X. Ying, Y. Wang, J. Liang, J. Yue, C. Xu, L. Lu, Z. Xu, J. Gao, Y. Du, Z. Chen, Angew. Chem. Int. Ed. 2014, 53, 12436-12440; Angew. Chem. 2014, 126, 12644-12648; b) D. Guarnieri, A. Falanga, O. Muscetti, R. Tarallo, S. Fusco, M. Galdiero, S. Galdiero, P. A. Netti, Small 2013, 9, 853-862.

[15] C. Velasco-Aguirre, F. Morales, E. Gallardo-Toledo, S. Guerrero, E. Giralt, E. Araya, M. J. Kogan, Int. J. Nanomed. 2015, 10, $4919-4936$.

Received: September 14, 2015

Published online: October 23, 2015 